\title{
Applications of Electron Microscopy to Complex Metal Hydrides
}

\author{
S.D. House*, I.M. Robertson*, A. Rockett*, L. Wang*, D.D. Johnson*, D.D. Graham ${ }^{\dagger}$ \\ * Dept. of Materials Science and Engineering, University of Illinois, Illinois, IL 61801 \\ * Ames Laboratory, Ames, Iowa, 50011 \\ ${ }^{\dagger}$ Intel Corporation, Portland, Oregon
}

Complex metal hydrides are potential hydrogen storage materials for transportation applications but to-date no system meets the requirements established by the Department of Energy. A key challenge is meeting the temperature and pressure window to ensure compatibility with fuel cells. This will require significant improvement in the thermodynamic and kinetic properties associated with the hydrogenation and dehydrogenation reactions. A major breakthrough was the discovery that additions of transition metals caused systems to be made reversible and in some cases improved the thermodynamics. In many candidate systems, there remains a lack of understanding of the location and form of these catalyst particles, and the morphological, structural and compositional changes that accompany hydrogenation and dehydrogenation. Scanning and transmission electron microscopy have the spatial resolution along with the techniques to provide insight to morphological, structural and compositional changes that occur during hydrogen cycles.

Examining complex metal hydrides in electron microscopes is challenging as some are damaged by exposure to the electron beam and some are air sensitive, which complicates sample preparation. Provided these challenges can be addressed, it is possible to use the capabilities of the electron microscope to explore the structure and composition of complex metal hydrides in the charged and discharged states and in the case where nanoporous structures are used to confine the metal hydride to determine the pore structure three-dimensionally.

In this talk, strategies for overcoming the challenges will be described and examples given to illustrate how electron microscope techniques have been used to learn how the catalyst particles are dispersed, how the composition varies during the synthesis of alane, and how electron tomography gives information about nano-confinement structures. For example, Figure 1 shows the dispersion of 3 wt $\% \mathrm{RuCl}_{3}$ and $\mathrm{TiCl}_{3}$ in $\mathrm{Ca}\left(\mathrm{BH}_{4}\right)_{2}$ following two cycles; the sample is in the dehydrided condition. The compositional maps show $\mathrm{Ru}$ remains mainly as isolated $\mathrm{RuCl}_{3}$, whereas the $\mathrm{Ti}$ is dispersed throughout [1]. Similar observations are made for other catalyst particles, raising the question about the operative mechanism. In studies of the morphology of the reversible, hydrided $\mathrm{AlH}_{3}$-TEDA adduct, it was found that the morphology and composition changes from the precursor and consists of electron transparent ribbons, agglomerates of activated $\mathrm{Al}$ particles, which contain $\mathrm{Ti}$ and isolated clusters of Al crystals [2]. Energy dispersive spectroscopy from fibers showed the presence of $\mathrm{Al}, \mathrm{C}, \mathrm{N}$, but not of $\mathrm{Ti}$; this analysis identified these as crystals of $\mathrm{AlH}_{3}$-TEDA. In contrast, in the $\mathrm{Al}$ particles $\mathrm{Ti}$ at levels of up to 0.4 at.\% was detected. This accumulation is significant as $\mathrm{Ti}$ is was introduced at a level of 0.1 at.\% $\mathrm{Ti}$, which suggests low levels of $\mathrm{Ti}$ are responsible for any catalytic effect. This finding could have important implications for understanding the role of catalytic species in other systems. These and other examples, including applications of electron tomography to explore the dispersion of catalyst particles and nano-confined structures will be described in detail in this talk. 


\section{References}

[1] Graham, D.D., Ph.D. Thesis, Materials Science and Engineering. 2009, Univ. of Illinois.

[2] Graham, D.D., et al., Location of Ti Catalyst in the Reversible AlH $\mathrm{Adduct}_{3}$ of Triethylenediamine. The Journal of Physical Chemistry C, 2010. 114(35): p. 15207-15211.

[3] This work was supported by DOE EERE through grant DE-FC36-05GO15064 and performed using the facilities in the Center for Microanalysis of Materials in the Frederick Seitz Materials Research Laboratory at the University of Illinois. IMR acknowledges support from the National Science Foundation while working at the Foundation.
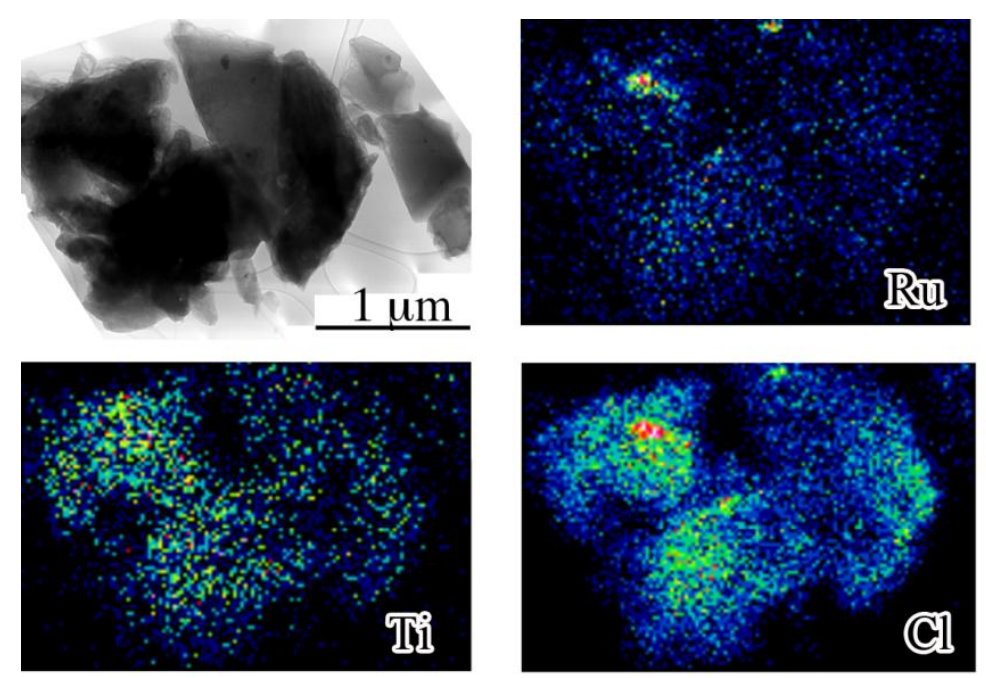

FIG 1 . Dispersion of Ti and $\mathrm{Ru}$ in 3 wt $\% \mathrm{RuCl}_{3}$ and $\mathrm{TiCl}_{3}$ doped $\mathrm{Ca}\left(\mathrm{BH}_{4}\right)_{2}$ following two cycles.
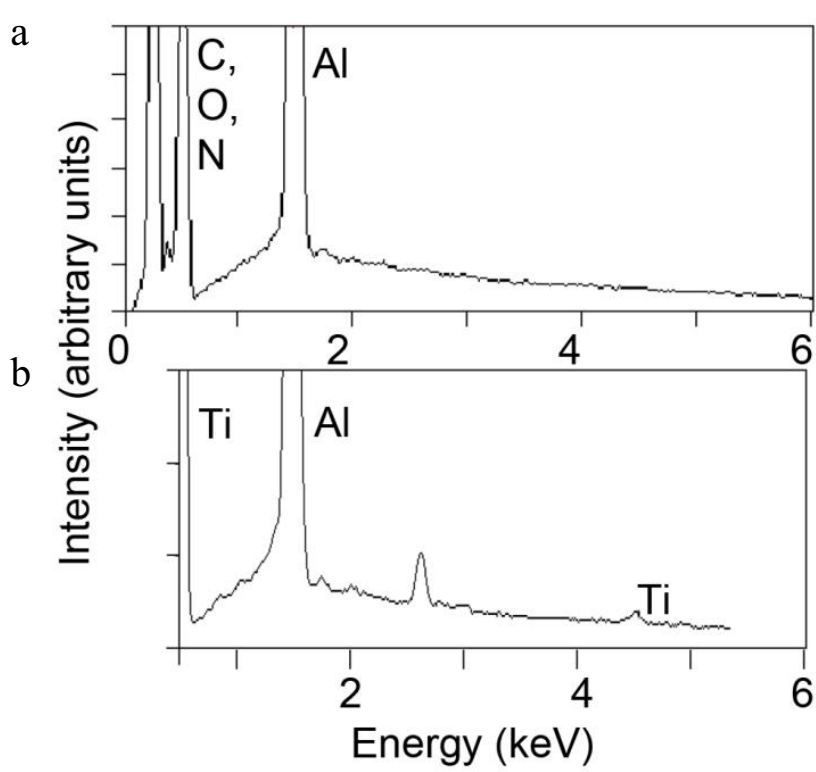
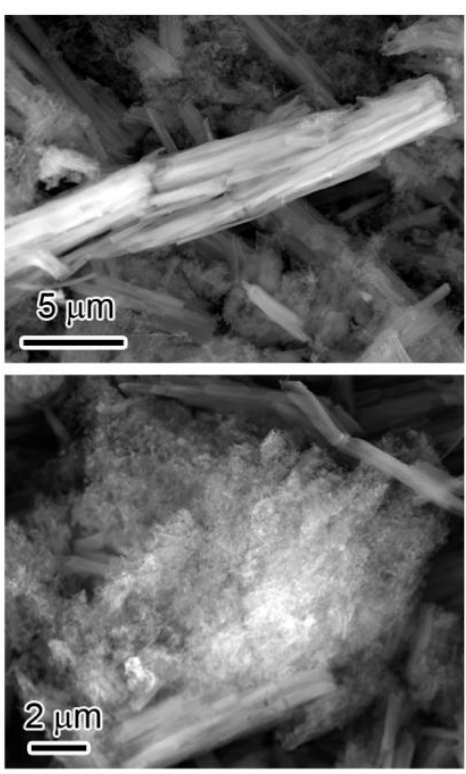

FIG 2. Composition, as determined by EDS, of the prominent morphological structures found in $\mathrm{AlH}_{3}$-TEDA samples. (a) shows the crystal and (b) the residual nodules. 